\title{
Subcutaneous ICD and ventricular tachycardia degeneration
}

\author{
Anna Mengoni $^{1 *}$, Erberto Carluccio ${ }^{1}$, Gianluca Zingarini ${ }^{2}$, Giuseppe Ambrosio ${ }^{1}$, Alessandra Tordini ${ }^{1}$, Paolo Biagioli ${ }^{1}$, David Giannandrea ${ }^{3}$ \\ and Claudio Cavallini ${ }^{2}$ \\ ${ }^{1}$ Cardiology and cardiovascular physiopathology, S. Maria della Misericordia Hospital, Perugia, Italy \\ ${ }^{2}$ Cardiology, Maria della Misericordia Hospital, Perugia, Italy \\ ${ }^{3}$ UO Neurology, USL Umbria 1 Centro Ictus - Gubbio e Gualdo Tadino Hospital, Italy
}

\begin{abstract}
Sudden cardiac death (SCD) remains a leading cause of cardiovascular mortality in the world. The trans-venous implantable cardioverter-defibrillator(TV-ICD) is the most effective approach for primary and secondary prevention of SCD. In response to the growing treatment complexity associated with long-term endocardial management, the subcutaneous implantable cardioverter-defibrillator ICD (SICD) was developed. We report a case in which S-ICD shocks changed a stable ventricular tachycardia (VT) to unstable VT o ventricular fibrillation (VF).
\end{abstract}

\section{Case report}

We reported the case of a 55 years old man admitted to our hospital because of arrhythmic storm and repeated shocks from a subcutaneous implantable cardioverter-defibrillator ICD (S-ICD). His clinical history was relevant for cardiomyopathy post-myocarditis (2011) with reduced left ventricular (LV) ejection fraction (EF, 40\%) due to hypoakinesia of infero-posterior wall and posterior interventricular septum, without significant coronary artery stenotic lesions at coronary angiography. In the same year, the patient received trans-venous cardioverter-defibrillator (TV-ICD) implant because of non-sustained ventricular tachycardia (VT) episodes during ambulatory ECG Holter monitoring. Subsequently, several appropriate anti-tachycardia pacing interventions (ATP) and shock-therapy occurred. In 2014, the patients developed endocarditis involving the device lead. This leads the device be explanted. Because of the high arrhythmic risk and the impossibility to wait for the end of entire antibiotic therapy, and taking into account the patient's choice, it was decided to not implant a new TV-ICD in the contralateral side. Therefore, a S- ICD was implanted. The device was programmed to recognize ventricular arrhythmias when 18 out of 24 consecutive sensed events exceed a pre-determined non-programmable detection limit zone; the shock impedances was set to $64 \mathrm{Ohm}$ and the shock energy 80 Joule, with standard shock polarity.

In September 2015 the patient was again admitted to the Hospital because of new arrhythmic storm and repeated shocks from a S-ICD. In the emergency room, he received lidocaine $100 \mathrm{mg}$ iv and amiodarone $300 \mathrm{mg}$ iv, without benefit. At the admission in the Intensive Care Unit, his blood pressure was 100/60 mmHg, heart rate190 beats/ min and oxygen saturation $98 \%$ in room air. Biochemical laboratory exams did not show any electrolytes imbalance. However, intravenous administration of magnesium sulphate, potassium and esmolol produced a significant attenuation of the arrhythmic events (Figure 1).

The S-ICD interrogation showed several VT episodes, effectively treated by device intervention (Figure 2, panel A). However, after the first S-ICD shocks, several episodes of degeneration of VT in ventricular fibrillation (VF) were also recorded (Figure 2, panel B), which were always interrupted by repeated further S-ICD's interventions. Therefore, because of repeating shocks, it was first decided to deactivate the S-ICD. Few days later, patients underwent radio frequency ablation of the ventricular arrhythmias, which was not fully therapeutically resolvent.

Therefore, the S-ICD was explanted and substituted by a traditional TV-ICD. During the subsequent 12 months follow-up, periodical ambulatory ICD interrogations showed several VT episodes, effectively treated with ATP (Figure 3).

\section{Discussion}

This dramatic case of arrhythmic storm, favourite by a device, underlies the importance to recognize shortcomings of any technology. Review of the literature reports this complication in about $4 \%$ of TVICD therapy triggered by ATP. However, nowadays no cases have been described for S-ICD has. Our patient apparently had not precipitant causes of VT (i.e. acute coronary syndrome or electrolytes imbalance); therefore, it is possible that dispersion of ventricular repolarisation and repeated shocks in the vulnerable period could have induced degeneration of VT [1].

The dispersion of ventricular repolarisation correlates closely with the vulnerable period to single electrical shocks and several studies have reported a link between the dispersion of repolarisation and the susceptibility to arrhythmias [2-4]. Non-uniform recovery of ventricular excitability plays an important role in the genesis of

Correspondence to: Anna Mengoni, Cardiology and cardiovascular physiopathology, S Maria della Misericordia Hospital, Perugia, Italy; Tel: 0755782394 .

Received: March 12, 2017; Accepted: April 04, 2017; Published: April 07, 2017 


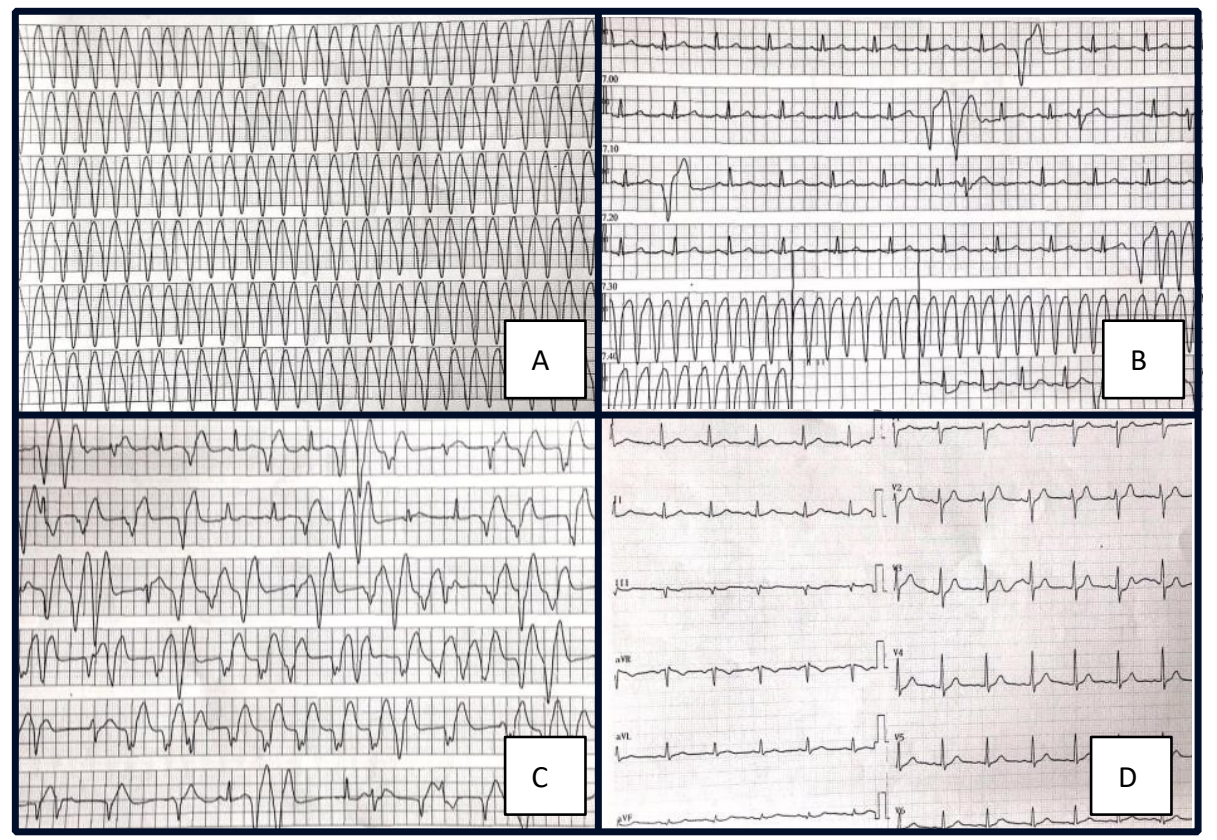

Figure 1. Panel A Telemetry: ventricular tachycardia. Panel B Telemetry:Sinus rhythm, ventricular beats, ventricular tachycardia interrupted by a DC-Shock. Panel C Telemetry: Sinus and Ventricular beats. Panel D Electrocardiogram: sinus rhythm, Q wave in DIII and aVF.

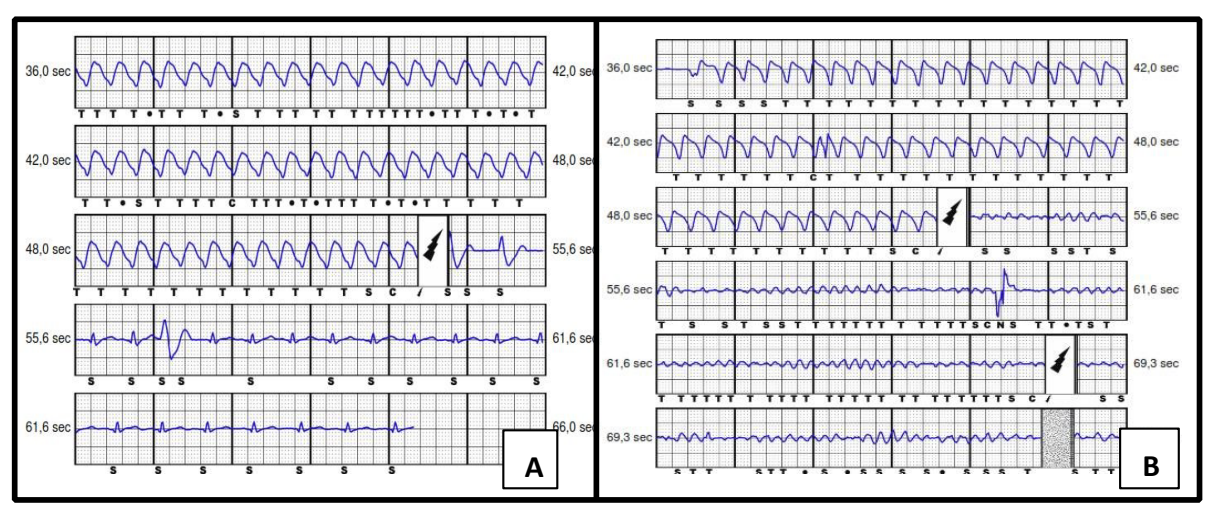

Figure 2. S-ICD control. Panel A: Ventricular tachycardia treated effectively with S-ICD shock. Panel B: Ventricular tachycardia degenerated in ventricular fibrillation after S-ICD shock and S-ICD shock not effectively on ventricular fibrillation.

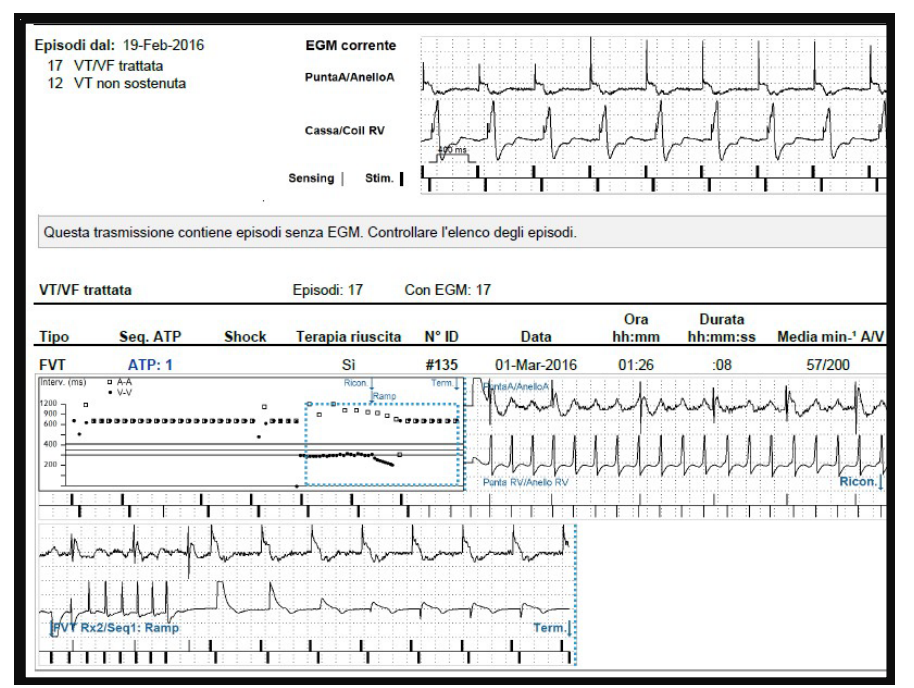

Figure 3. A remote monitoring shows 12 episodes of non sustained ventricular tachycardia and 17 episodes of sustained ventricular tachycardia treated effectively with anti tachycardia pacing. ventricular arrhythmia; in fact, shock acting in the context of tissue with heterogeneous repolarisation may induce additional action potentials in regions with short repolarisation times and prolonged action potentials in regions with long repolarisation times [5]. Overall, this may facilitate re-entry of multiple activation wave fronts and induction of ventricular fibrillation.

In our patients, the dispersion of ventricular depolarisation is likely to depend on the pathophysiological substrate (fibrosis postmyocarditis).

S-ICD lack of an anti-tachycardia pacing function.

However, although in our patient several VT episodes were effectively terminated by anti tachycardia pacing, we decided to implant S-ICD before discharge for several reasons:

1) As demonstrated by the START trial, both TV-ICD and S-ICD can effectively detect and treat ventricular arrhythmias [6]

2) many episodes of non-sustained VT, although being treated by ATP, generally are likely to terminate spontaneously [7] 
3) ATP therapy could also be harmful by converting a stable VT to an unstable VT or VF. Therefore, at the moment, an ideal device does not exist; both TV-ICD and S-ICD are similar for efficacy and safety and the choice may be done only on clinical considerations.

\section{References}

1. Shibata N, Chen PS, Dixon EG, et al. (1988) Influence of shock strength and timing on induction of ventricular arrhythmias in dogs. Am J Physiol 255:H891-H901. This finding concurs with the hypothesis that the vulnera- bility of the heart relates to the late repolarisation phase of the action potential

2. Wiggers CJ, Wegren R (1940) Ventricular fibrillation due to single local- ized induction in condenser shock supplied during the vulnerable phase of ventricular systole. $A m J$ Physiol 128:500-505.

3. Brugada J, Brugada P, Bcersma L, et al. (1991) On the mechanisms of ventricular tachycardia acceleration during programmed electrical stimulation. Circulation 83: 1621-1629.
4. Taggart P, Sutton P, Lab M, Dean J, Harrison F (1990) Interplay between adrenaline and interbeat interval on ventricular repolarisation in intact heart in vivo. Cardiovasc Res 24:884-895.

5. Franz MR, Swerdlow CD, Liem LB, Schaefer J (1988) Cycle length dependence of human action potential duration in vivo. Effects of single extrastimuli, sudden sustained rate acceleration and deceleration, and different steady-state frequencies. $J$ Clin Invest 82: 972-979. [Crossref]

6. Gold MR, Theuns DA, Knight BP, Sturdivant JL, Sanghera R, et al. (2012) Headto-head comparison of arrhythmia discrimination performance of subcutaneous and transvenous ICD arrhythmia detection algorithms: the START study. $J$ Cardiovasc Electrophysiol 23: 359-366. [Crossref]

7. Moss AJ, Schuger C, Beck CA, Brown MW, Cannom DS, et al. (2012) Reduction in inappropriate therapy and mortality through ICD programming. $N$ Engl J Med 367: 2275-2283. [Crossref]

Copyright: (C2017 Mengoni A. This is an open-access article distributed under the terms of the Creative Commons Attribution License, which permits unrestricted use, distribution, and reproduction in any medium, provided the original author and source are credited. 